\title{
Outdoor Growth Characterization of an Unknown Microalga Screened from Contaminated Chlorella Culture
}

\author{
Shuhao Huo, ${ }^{1,2,3}$ Changhua Shang, ${ }^{2,4}$ Zhongming Wang, ${ }^{2}$ Weizheng Zhou, ${ }^{2}$ \\ Fengjie Cui, ${ }^{1}$ Feifei Zhu, ${ }^{1}$ Zhenhong Yuan, ${ }^{2}$ and Renjie Dong ${ }^{3}$ \\ ${ }^{1}$ School of Food and Biological Engineering, Jiangsu University, Zhenjiang 212013, China \\ ${ }^{2}$ Guangzhou Institute of Energy Conversion, Chinese Academy of Sciences, Guangzhou 510640, China \\ ${ }^{3}$ College of Engineering, China Agricultural University, Beijing 100083, China \\ ${ }^{4}$ Institute of New Energy and New Materials, South China Agricultural University, Guangzhou, China \\ Correspondence should be addressed to Zhongming Wang; wangzm@ms.giec.ac.cn and Renjie Dong; rjdong@cau.edu.cn
}

Received 17 March 2016; Revised 29 July 2016; Accepted 14 December 2016; Published 5 March 2017

Academic Editor: Atanas Atanassov

Copyright (C) 2017 Shuhao Huo et al. This is an open access article distributed under the Creative Commons Attribution License, which permits unrestricted use, distribution, and reproduction in any medium, provided the original work is properly cited.

Outdoor microalgae cultivation process is threatened by many issues, such as pest pollution and complex, changeable weather. Therefore, it is difficult to have identical growth rate for the microalgae cells and to keep their continuous growth. Outdoor cultivation requires the algae strains not only to have a strong ability to accumulate oil, but also to adapt to the complicated external environment. Using $18 \mathrm{~S}$ rRNA technology, one wild strain Scenedesmus sp. FS was isolated and identified from the culture of Chlorella zofingiensis. Upon contamination by Scenedesmus sp., the species could quickly replace Chlorella zofingiensis G1 and occupy ecological niche in the outdoor column photobioreactors. The results indicated that Scenedesmus sp. FS showed high alkali resistance. It also showed that even under the condition of a low inoculum rate $\left(\mathrm{OD}_{680}, 0.08\right)$, Scenedesmus sp. FS could still grow in the outdoor raceway pond under a high alkaline environment. Even under unoptimized conditions, the oil content of Scenedesmus sp. FS could reach more than $22 \%$ and $\mathrm{C} 16-\mathrm{C} 18$ content could reach up to $79.68 \%$, showing that this species has the potential for the biodiesel production in the near future.

\section{Introduction}

Due to the exorbitant cost input into nutritive salts such as chemical fertilizers and high energy consumption in microalgae harvest, the microalgal biodiesel has not yet been successfully applied in commercial production [1-4]. Utilizing sunlight to magnify the cultivation of microalgae under outdoor conditions is an effective way to reduce the cost of microalgal cultivation. The current studies on energy microalgae are mainly carried out at indoor labs as there are many difficulties in outdoor cultivation [5-7]. In outdoor cultivation, the microalga of interest is often vulnerable to contamination with viruses, bacteria, fungi, insect pupae, rotifers, protozoa, or other unwanted algal species. Among them, rotifers and protozoa are the two organisms that are able to seize ecosystem niche quickly due to their small body, simple structure, and fast reproduction speed. They prey on microalgae cells, resulting in a great reduction of microalgae cell concentration, and exceedingly threatening the microalgae production. Meanwhile, the complex and changeable outdoor weather conditions make the cultivation of microalgae have an uneven growth rate and thus production is often difficult to be carried out [8-10].

Excellent microalgal strain is crucial to the realization of microalgal biodiesel production. Outdoor cultivation requires the selected algal strain not only to have a strong ability to accumulate oil but also to adapt to the external environment. The lipid accumulation of microalgae can be improved by changing the element content in the culture medium such as the deprivation of nitrogen, phosphorus, or other elements, while the outdoor adaptability of microalgae is more difficult to be improved in a short time. Through the amplification of $18 \mathrm{~S}$ rRNA sequences of unknown algal strains, the wild microalgal strain screened from contaminated Chlorella zofingiensis G1 in an outdoor column photobioreactor was preliminarily identified in this 


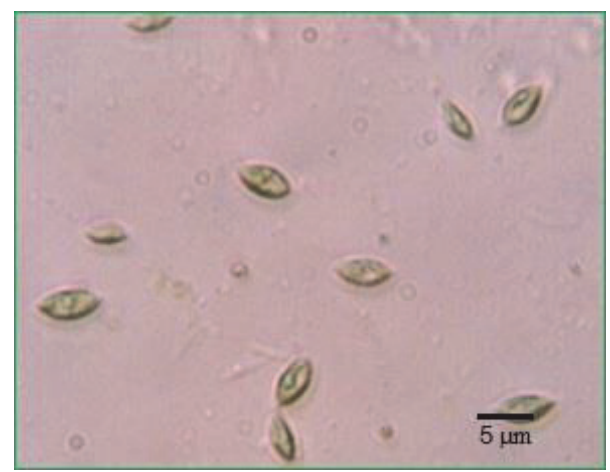

FIGURE 1: The wild microalgal strain in the outdoor pond observed with light microscope at $\times 400$ magnification.

paper. Chlorella zofingiensis G1 could be quickly replaced by the wild microalgal strain (Figure 1), which could occupy ecological niche quickly, showing high alkali resistance and antipollution performances. The wild microalgal fronds are unicellular ellipse in shape with a smooth surface, and the cell size is approximately $5.0-7.0 \mu \mathrm{m}$ in length and $2.0-4.0 \mu \mathrm{m}$ in width under light microscope. At the same time, this paper also examined the possibility for large-scale cultivation in the outdoor raceway pond.

\section{Materials and Methods}

2.1. Materials. Colonies of microalgae were isolated from contaminated Chlorella zofingiensis G1 in an outdoor photobioreactor in the district of Sanshui, Foshan city, China $\left(23^{\circ} 03^{\prime} \mathrm{N}-112^{\circ} 09^{\prime} \mathrm{E}\right)$. The isolated algal cells were cultured and maintained in a BG11 medium [11] at $25^{\circ} \mathrm{C}$ under continuous illumination by cool-white fluorescent lamps (light intensity: 2000 lux) in a $500 \mathrm{~mL}$ Erlenmeyer flask. Aeration and mixing were achieved by the sparging air with $6.0 \% \mathrm{CO}_{2}$ through a glass-filter, which was inserted to the bottom of the reactor and the flow rate of gas was $0.5 \mathrm{vvm}$, regulated by the gas flow meter (Model G, Aalborg Instruments \& Controls, Inc., Orangeburg, NY, USA). The temperature of the culture media was $25 \pm 1^{\circ} \mathrm{C}$, regulated by the room air conditioner (Gree Electric Appliances Inc., Zhuhai, Guangdong, China). After 6 days of cultivation, when the cells were in the logarithmic phase, the cultures were used for outdoor experiments.

A $40 \mathrm{~L}$ vertical tubular outdoor photobioreactor $(8.7 \mathrm{~cm} \times$ $160 \mathrm{~cm}=$ diameter $\times$ height $)$ was used to cultivate the abovementioned strain as seed cultures for the outdoor raceway pond amplification cultivation.

rTaq, pMD18-T, and T4 DNA Ligase were obtained from Takara Biotech Co., Ltd., China. EasyPure Quick Gel Extraction Kit was obtained from Beijing TransGen Biotech Co., Ltd., China. The nucleotide sequences of these primers (Table 1) were synthesized by Sangon Biotech Co., Ltd., China. DNA sequencing was analyzed by Shanghai Life Technologies Corporation, China. The primers NS1 and NS8 were used to clone the $18 \mathrm{~S}$ rRNA sequence of microalgae. The primers M13 (-40) Forward and M13 Reverse were used to clone the
TABLE 1: Oligonucleotide primers used in this work.

\begin{tabular}{lr}
\hline Primer & Sequence $\left(5^{\prime} \rightarrow 3^{\prime}\right)$ \\
\hline NS1 & GTAGTCATATGCTTGTCTC \\
NS8 & TCCGCAGGTTCACCTACGGA \\
M13 $(-40)$ Forward & GTTTTCCCAGTCACGAC \\
M13 Reverse & CAGGAAACAGCTATGAC \\
\hline
\end{tabular}

inserted gene fragment in pMD18-T and confirm the success of TA cloning.

\subsection{Identification Methods}

2.2.1. Microscopic Observation. After shaking evenly, a drop of $0.05 \mathrm{~mL}$ microalgae sample was dripped onto the slide, and the sample was covered by glass $(18 \times 24 \mathrm{~mm})$ and observed with polarizing microscope from Nikon Instruments Eclipse LV100 POL at $\times 400$ magnification.

\subsubsection{Isolation of Genomic DNA of the Wild Microalgal Strain.} Wild microalgal strain was harvested during logarithmic phase after 3-4 days of cultivation in a BG11 medium, frozen in liquid nitrogen, and grounded using a pestle and mortar. The genomic DNA was isolated by the CTAB method [12].

\subsubsection{PCR Amplification of $18 S \mathrm{rRNA}$ from the Wild Microalgal} Strain. The $18 \mathrm{~S}$ rRNA was amplified by PCR using the NS1 and NS8 universal primers as shown in Table 1 and the genomic DNA was used as a template for PCR amplification. PCR amplification was carried out in $0.2 \mathrm{~mL}$ tubes. The PCR mixture included 10x PCR Buffer $\left(\mathrm{Mg}^{2+}\right.$ plus) $5 \mu \mathrm{L}$, dNTPs $(2.5 \mathrm{mM}) 4 \mu \mathrm{L}, \mathrm{NS} 1(20 \mu \mathrm{M}) 1 \mu \mathrm{L}$, NS8 $(20 \mu \mathrm{M}) 1 \mu \mathrm{L}$, genomic DNA $1 \mu \mathrm{L}$, rTaq $(5 \mathrm{U} / \mu \mathrm{L}) 0.2 \mu \mathrm{L}$, and deionized water $37.8 \mu \mathrm{L}$, with a total volume of $50 \mu \mathrm{L}$. Amplification conditions were as follows: 30 cycles at $94^{\circ} \mathrm{C}$ for $30 \mathrm{~s}, 50^{\circ} \mathrm{C}$ for $30 \mathrm{~s}$, and $72^{\circ} \mathrm{C}$ for $2 \mathrm{~min}$, followed by a final extension at $72^{\circ} \mathrm{C}$ for $5 \mathrm{~min}$. PCR products were fractionated in $2 \%(\mathrm{w} / \mathrm{v})$ agarose gels and stained with ethidium bromide.

\subsubsection{The Purification, Ligation, and Transformation of PCR} Product. The PCR product was recovered using EasyPure Quick Gel Extraction Kit (Trans, Beijing), according to the instruction book. The amplification products were ligated into pMD18-T vector (Takara) and then transformed and sequenced, according to standard procedures described by Sambrook et al. [13].

2.2.5. The Identification of Positive Transformants. Amplified fragments with resistance to ampicillin were picked from the medium, they were cultured in $3 \mathrm{~mL}$ LB liquid medium at $150 \mathrm{rpm}$ for about $20 \mathrm{~h}$, and the colony was identified through PCR using $1 \mu \mathrm{L}$ culture. Transformed E. coli DH5 $\alpha$ were picked from the medium containing $100 \mu \mathrm{g} / \mathrm{mL}$ ampicillin. The PCR mixture included 10x PCR Buffer $\left(\mathrm{Mg}^{2+}\right.$ plus) $5 \mu \mathrm{L}$, dNTPs $(2.5 \mathrm{mM}) 4 \mu \mathrm{L}, \mathrm{M} 13(-40)$ Forward $(20 \mu \mathrm{M}) 1 \mu \mathrm{L}$, M13 Reverse $(20 \mu \mathrm{M}) 1 \mu \mathrm{L}$, liquid culture $1 \mu \mathrm{L}$, rTaq $(5 \mathrm{U} / \mu \mathrm{L})$ $0.2 \mu \mathrm{L}$, and deionized water $37.8 \mu \mathrm{L}$, with a total volume of 
TABLE 2: The quality of mountain spring water*

\begin{tabular}{lc}
\hline Elements & Content $(\mu \mathrm{g} / \mathrm{L})$ \\
\hline $\mathrm{Mn}$ & $5.99 \pm 3.46$ \\
$\mathrm{Fe}$ & $22.37 \pm 17.52$ \\
$\mathrm{P}$ & $128.28 \pm 1.50$ \\
$\mathrm{Si}$ & $3481.00 \pm 9.90$ \\
$\mathrm{Na}$ & $28.27 \pm 0.64$ \\
$\mathrm{~K}$ & $160.05 \pm 6.12$ \\
$\mathrm{Mg}$ & $1174.60 \pm 9.33$ \\
$\mathrm{Zn}$ & $26.84 \pm 4.02$ \\
$\mathrm{Ca}$ & $1979.60 \pm 12.16$ \\
$\mathrm{Mo}$ & n. d. \\
$\mathrm{Cu}$ & n. d. \\
\hline
\end{tabular}

* Note: average values of water samples collected in two different locations; n.d.: not detected.

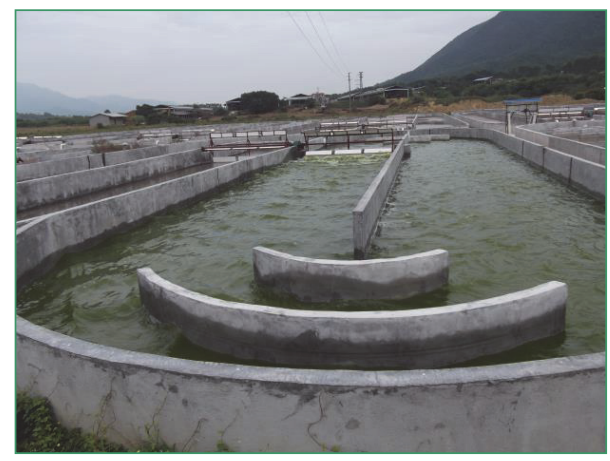

FIGURE 2: Photograph of outdoor ponds for wild microalgae cultivation $(8 \mathrm{~m} \times 50 \mathrm{~m})$.

$50 \mu \mathrm{L}$. Amplification conditions were as follows: 30 cycles at $94^{\circ} \mathrm{C}$ for $30 \mathrm{~s}, 46^{\circ} \mathrm{C}$ for $30 \mathrm{~s}$, and $72^{\circ} \mathrm{C}$ for $2 \mathrm{~min}$, followed by a final extension at $72^{\circ} \mathrm{C}$ for $5 \mathrm{~min}$. PCR products were size fractionated in $2 \%(\mathrm{w} / \mathrm{v})$ agarose gels and stained with ethidium bromide. Volume of $1.5 \mathrm{~mL}$ culture was selected for sequencing analysis (Life Technology, Shanghai).

2.2.6. Lipid Content and Fatty Acid Composition Analysis. Bigogno's method (2002) was applied to quantify the amount of total lipid content [14]. Fatty acid composition analysis was carried out by the saponification reaction with the participation of base catalyst [15].

\subsection{Outdoor Raceway Pond Cultivation of the Wild Microal-} gae. The outdoor raceway ponds (brick cement ponds) were located in Sanshui district, Foshan city, China $\left(23^{\circ} 03^{\prime} \mathrm{N}-\right.$ $112^{\circ} 09^{\prime} \mathrm{E}$ ). The ponds were $50 \mathrm{~cm}$ in height, $8 \mathrm{~m}$ in width, and $50 \mathrm{~m}$ in length from north to south with semicircular arc at both ends and had average of $23-25 \mathrm{~cm}$ depth of water (Figure 2). Paddle wheel device was installed to circulate the pool liquid, which was operated at about $10 \mathrm{~cm}$ depth and rotated at the speed of $15 \mathrm{rpm}$. Cultivation water was mountain spring water (Table 2). Nutrient composition was specified in Table 2. A large amount of chemical fertilizer was added to the outdoor raceway pond, including $300 \mathrm{mg} / \mathrm{L}$ of $\mathrm{CO}$

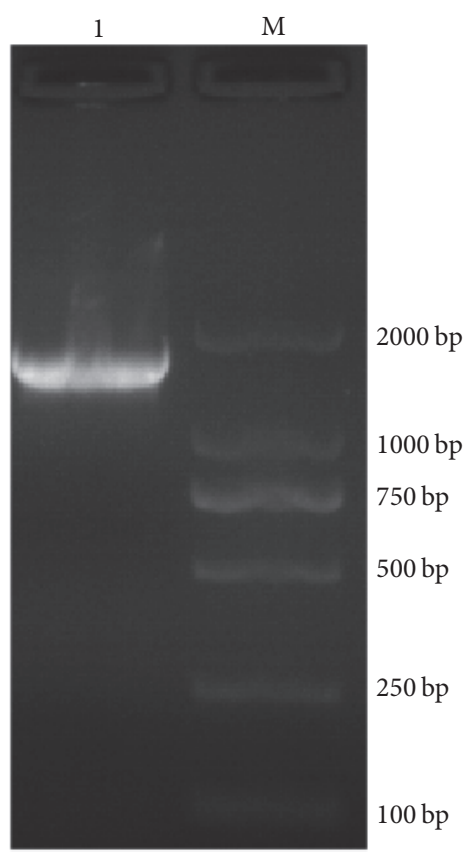

FIGURE 3: Genomic DNA from wild microalgal strains (left) and amplification results of $18 \mathrm{~S}$ rRNA of wild microalgal strain (1: 18S rRNA band; M: DL2000 DNA marker) (right).

$\left(\mathrm{NH}_{2}\right)_{2}, 60 \mathrm{mg} / \mathrm{L}$ of $\mathrm{KH}_{2} \mathrm{PO}_{4}$, and $60 \mathrm{mg} / \mathrm{L}$ of $\mathrm{MgSO}_{4}$. The initial optical density $\left(\mathrm{OD}_{680}\right)$ of the culture was controlled between 0.3 and 0.5 when appropriate amount preculture broths were inoculated into the $25 \mathrm{~m}$ long small pond during the logarithmic phase. In the process of cultivation, the $\mathrm{pH}$ value was not adjusted. The $\mathrm{pH}$, temperature, and light intensity were recorded 4 times a day $(8: 00,11: 00,14: 00$, and 17:00). The biomass concentration was determined by measuring the $\mathrm{OD}_{680}$ value of the sample in the pond at 17:00. After 7 days of cultivation, the wild microalgae were harvested.

\section{Results and Discussion}

3.1. Wild Algae Identification Using $18 S$ rDNA Technology. The CTAB method was used to extract the genomic DNA of wild microalgae strains. The results in Figure 3 showed that the genomic DNA sample was complete and no degradation phenomenon was found in $1 \%(\mathrm{w} / \mathrm{v})$ agarose gels electrophoresis stained with ethidium bromide.

By using universal primers NS1 and NS8 and the wild algae genomic DNA as a template for PCR amplification, a $1767 \mathrm{bp}$ band was obtained, as shown in Figure 3. A total of 12 positive transformants of $E$. coli DH5 $\alpha$ were identified using primers M13 (-40) Forward and M13 Reverse using $1 \mu \mathrm{L}$ culture as template. Electropherogram of PCR products was from 12 positive transformants of E. coli DH5 $\alpha$ containing recombinant pMD18-T. The bands were identified by electrophoresis. As shown in Figure 4, the results suggested that all 18S rRNA fragments with approximate length of $2 \mathrm{~kb}$ were successfully connected to the pMD18-T vector. The 12 positive transformants were named as $18 \mathrm{~S}-1 \sim 18 \mathrm{~S}-12$. Of them, 


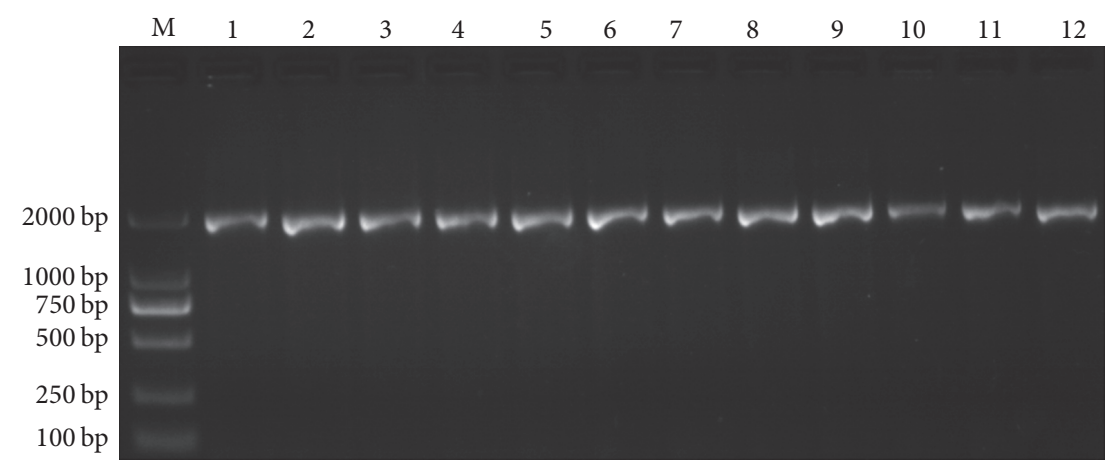

FIGURE 4: Electropherogram of PCR products from 12 positive transformants, carrying pMD18-T in which the $18 \mathrm{~S}$ rRNA gene sequence of the wild alga was cloned. M13 primers were used for identification.

\begin{tabular}{|c|}
\hline \\
\hline TCATTA \\
\hline GA \\
\hline 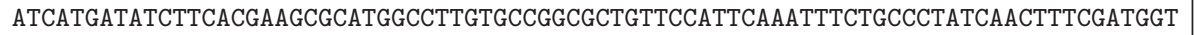 \\
\hline AGGATAGAGGCCTACCATGGTGGTAACGGGTGACGGAGGATTAGGGTTCGATTCCGGAGAGGGAGCCTGAGAAACG \\
\hline GCTACCACATCCAAGGAAGGCAGCAGGCGCGCAAATTACCCAATCCTGATACGGGGAGGTAGTGACAATAAATAACA \\
\hline ATACCGGGCATTTCATGTCTGGTAATTGGAATGAGTACAATCTAAATCCCTTAACGAGGATCCATTGGAGGGCAAGTCT \\
\hline GGTGCCAGCAGCCGCGGTAATTCCAGCTCCAATAGCGTATATT" \\
\hline CGGGTGGGTTCTAGCGGTCCGCCTATGGTGAGTACTGCTATGGCCTTCCTTTCTGTCGGGGACGGGCTTCTGGGCTTC \\
\hline ACTGTCCGGGACTCGGAGTCGACGTGGTTACTTTGAGTAAATTAGAGTGTTCAAAGCAGGCTTACGCCAGAATACTTT \\
\hline AGCATGGAATAACACGATAGGACTCTGGCCTATCTTGTTGGTCTGTAGGACCGGAGTAATGATTAAGAGG \\
\hline AGAGGTGAAATTCTTGGATTTATGAAAGACG \\
\hline GATGTTTTCAT \\
\hline GCCGACTAGGGATTGGCGAATGTTTTTTTAATGACTTCGCCAGCACCTTATGAGAAATCAAAGTTTTTGGG \\
\hline GGGAGTATGGTCGCAAGGCTGAAACTTAAAGGAATTGACGGAAGGGCACCACCAGGCGTGGAGCCTGCGGCTTAAT \\
\hline TTGACTCAACACGGGAAAACTTACCAGGTCCAGACATAGTGAGGATTGACAGATTGAGAGCTCTTTCTTGATTCTATG \\
\hline GGTGGTGGTGCATGGCCGTTCTTAGTTGGTGGGTTGCCTTGTCAGGTTGATTCCGGTAACGAACGAGACCTCAGCCT \\
\hline ITAGTCTCAGTTGCTTTTTGCAGCTGGCTGACTTCTTAGAGGGACTATTGGCGTTTAGTCAATGGAAG \\
\hline GCAATAACAGGTCTGTGATGCCCTTAGATGTTCTGGGCCGCACGCGCGCTACACTGATGCATTCAACAA \\
\hline TGACCGAAG \\
\hline AATGCCTAGTAAGCGCAAGTCATCAGCTTGCGTTGATTACGTCCCTGCO \\
\hline GATTGGGTGTGCTGGTGAAGTGTTCGGATTGGCAGCTTAGGGTGGCAACACCTCAGGTCTGCC \\
\hline ACCCTCCCACCTAGAGGAAGGAGAAGTCGTAACAAGGTTTCCGTAGGTGAACCTGCGGAA \\
\hline
\end{tabular}

FIGURE 5: $18 \mathrm{~S}$ rDNA gene sequence of the wild strain.

18S-1 18S-5 were selected for sequencing and the results were completely consistent (analyzed by Shanghai Life Biotech Co., Ltd.).

The 18S rRNA gene sequence amplified from this strain is $1767 \mathrm{bp}$ in length (Figure 5), which showed similarities with other known sequences from green algae based on the BLAST $n$ results, with homology above $99 \%$ to Scenedesmus obliquus and Scenedesmus acutus. The phylogenetic analysis indicated that this strain has a close relationship with Scenedesmus sp., named Scenedesmus sp. FS (Figure 6). The sequences of $18 \mathrm{~S}$ rRNA gene (fragment) of those microalgae in NCBI GenBank were as follows: Scenedesmus obliquus: FR865738.1; Scenedesmus acutus:
AJ249512.1; Scenedesmus subspicatus: AJ249514.1; Neochloris vigenis: M74496.1; Chlorella vulgaris strain CCAP 211/11F: AY591515.1; Chlorococcum oleofaciens: KM020101.1; Chlamydomonas sp. A-SIO: AF517100.1; Chlamydomonas segnis: U70593.1; Chlorococcum hypnosporum: U41173.1; Chlamydomonas cribrum: LC086333.1; Chlamydomonas mexicana: AF395434.1; Scenedesmus sp. FS: KY268297.

3.2. Cultivation of Scenedesmus sp. FS in the Outdoor Raceway Pond. As shown in Figure 7, the optical density of inoculum was very low $\left(\mathrm{OD}_{680}, 0.08\right)$, and the raceway pond had a large surface area to volume ratio, which could inhibit the 


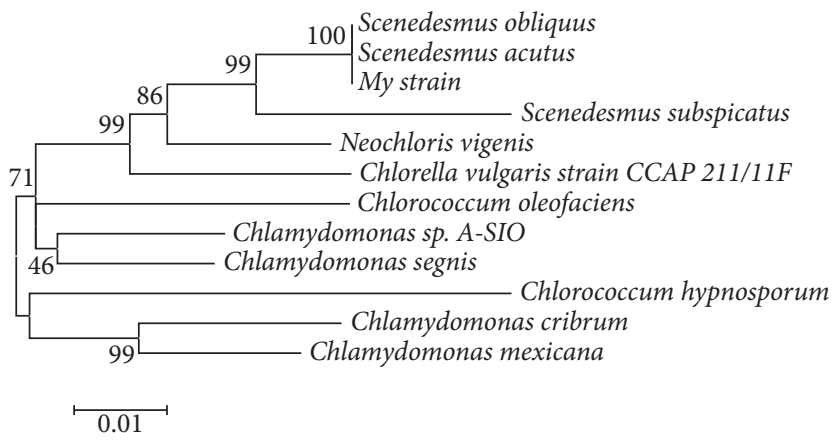

Figure 6: Phylogenetic tree constructed based on the 18S rRNA gene sequences of 11 strains of green algae and the experimental microalga (Bootstrap values are indicated as percentages at the nodes).

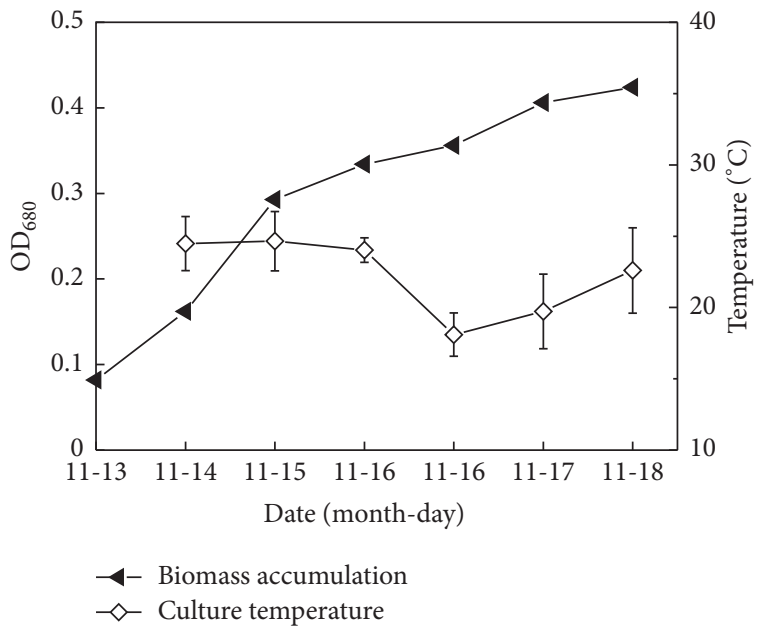

FIGURE 7: Growth curve and fluctuation of culture temperature (an average value from 8:00 am to 5:00 pm each day).

growth of single cells at the initial phase due to strong light. But in fact Scenedesmus sp. FS was still able to adapt to the environment of outdoor raceway pond. The growth and metabolism of Scenedesmus sp. FS was active, and the algae did not experience the lag phase and directly got into the logarithmic phase with a rapid growth rate.

In the experiment, light intensity and temperature in the outdoor changed obviously, and the highest light intensity reached more than $1500 \mu \mathrm{mol} /\left(\mathrm{m}^{2} \cdot \mathrm{s}\right)$, and water temperature ranged from 18.1 to $24.7^{\circ} \mathrm{C}$. Sánchez et al. (2008) reported that the optimal growth temperature of Scenedesmus almeriensis was $35^{\circ} \mathrm{C}$, and it could still survive at $48^{\circ} \mathrm{C}$. This algae could tolerate high light intensity $\left(1625 \mu \mathrm{mol} /\left(\mathrm{m}^{2} \cdot \mathrm{s}\right)\right)$ and could also accumulate $0.55 \%$ (wt\%) lutein under this light intensity [16]. In this experiment, the wild algae Scenedesmus sp. FS was also able to tolerate similar light intensity. Additionally, the wild alga was able to grow under high alkaline condition with $\mathrm{pH}$ values between 9.0 and 9.7 (Figure 8). The dissolved oxygen changed between 9.5 and $10.9 \mathrm{mg} / \mathrm{L}$ daily, which was relatively stable. It can be seen that the wild algae Scenedesmus sp. FS could well adapt to the outdoor conditions in the process of cultivation. The pest pollution and other alien invasive algae in this experiment were not significant, which indicates the

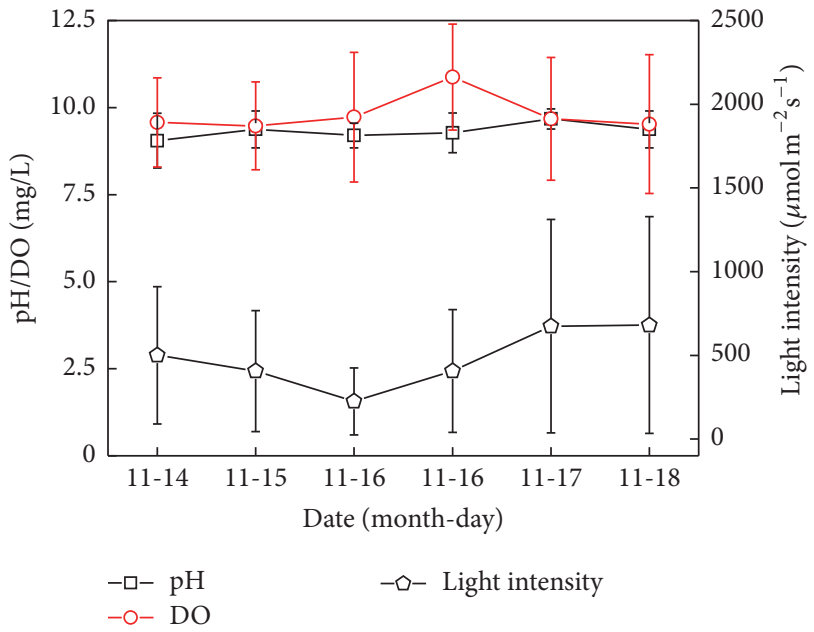

Figure 8: The fluctuation of light intensity, $\mathrm{pH}$, and dissolved oxygen (DO) (the average value in the range of 8:00 am 5:00 pm) under outdoor conditions.

scale-up potential of the wild algae under outdoor culture conditions. In future studies, outdoor cultivation conditions need further optimization to obtain larger biomass and higher oil yield rate.

3.3. Oil Content and Fatty Acid Composition of Scenedesmus sp. FS. Numerous studies have shown that nitrogen deficiency can promote accumulation of oil in microalgae [17]. Goldberg and Cohen found that phosphorus deficiency could also significantly promote oil accumulation of Monodus subterraneus [18]. Under these conditions, the fixed $\mathrm{CO}_{2}$ of algal cells would be converted with priority into lipid or carbohydrates, rather than proteins [8]. In normal culture condition, fatty acid synthesized was mainly used in the synthesis of membrane sugar-based glyceride and phospholipids, which accounted for about $5-20 \%$ of the dry weight of the algae cells. Under the nitrogen deficiency and other adverse environmental conditions, algae cell would change the oil synthesis route, beginning to accumulate neutral fat of about $20-50 \%$ of the dry weight, with triacylglycerol (TAG) as the main component. After 6 days of cultivation, the total nitrogen and phosphorus concentration in the pond were 
TABLE 3: Fatty acid composition (wt\%) of the wild Scenedesmus sp. FS cells.

\begin{tabular}{lc}
\hline Fatty acid & wt $\%$ \\
\hline C16:0 & 24.55 \\
C16:1 & 2.74 \\
C18:0 & 2.56 \\
C18:2 & 40.64 \\
C18:3 & 9.19 \\
C20:1 & 4.64 \\
C20:2 & 4.53 \\
C22:1 & 4.29 \\
C24:1 & 2.45 \\
\hline
\end{tabular}

$70.8 \pm 2.8 \mathrm{mg} / \mathrm{L}$ and $6.0 \pm 0.1 \mathrm{mg} / \mathrm{L}$, respectively, indicating a sufficient supply of nitrogen and phosphorus. Oil content in the algal cells increased slowly and oil content of Scenedesmus sp. FS was not high with fatty acid composition $22.0 \pm 1.9 \%$ of the algal weight.

The compositions of fatty acid methyl esters (FAMEs) were shown in Table 3. The microalgal lipids mainly contained FAMEs with 16-18 carbons with a high cetane number. These lipids show better fuel properties and low temperature performance and were considered suitable for sustainable biodiesel production [19]. The content of FAMEs C16-C18 in the wild strain Scenedesmus sp. FS reached $79.68 \%$, with C16:0 24.55\% and C18:2 40.64\%, showing good potential for development of biodiesel.

\section{Conclusion}

Using $18 \mathrm{~s}$ rDNA molecular technology, the dominant microalgae strain screened from contaminated Chlorella zofingiensis G1 in an outdoor photobioreactor was identified. The isolated species belonged to Scenedesmus genus, which was named Scenedesmus sp. FS. Under the conditions of a low concentration inoculation, this strain still had good alkali resistance and robust adaption to the stress of the outdoor environment. It had great potential as a large-scale cultivation strain for biodiesel production. Further research would be focused on the optimization of the culture conditions and the use of random mutagenesis technology, directed evolution method, or other technical means to obtain higher biomass and oil yield of the alga Scenedesmus sp. FS.

\section{Competing Interests}

The authors declare that they have no competing interests.

\section{Authors' Contributions}

Shuhao Huo, Changhua Shang, and Weizheng Zhou wrote the main part of the paper and performed the experiments. Zhongming Wang conceived the experiments. Others read and approved the manuscript. Shuhao Huo and Changhua Shang contributed equally to this work.

\section{Acknowledgments}

This research was funded by the National Natural Science Foundation of China (21506084, 21406093), the China Postdoctoral Science Foundation (2015T80502), the Natural Science Foundation of Jiangsu Province (BK20140540), the key Laboratory of Renewable Energy, Chinese Academy of Sciences (no. y507k11001), A Project Funded by the Priority Academic Program Development of Jiangsu Higher Education Institutions (PAPD), and the Training Project of the Young Core Instructor of Jiangsu University.

\section{References}

[1] Y. Chisti, "Biodiesel from microalgae," Biotechnology Advances, vol. 25, no. 3, pp. 294-306, 2007.

[2] J. Sheehan, T. Dunahay, J. Benemann, and P. Roessler, "A look back at the U.S. Department of Energy's aquatic species program: biodiesel from algae," NREL Report TP-580-24190, NREL, Golden, Colo, USA, 1998.

[3] L. Zhu, "Microalgal culture strategies for biofuel production: a review," Biofuels, Bioproducts and Biorefining, vol. 9, no. 6, pp. 801-814, 2015.

[4] L. Zhu, S. Huo, and L. Qin, "A microalgae-based biodiesel refinery: sustainability concerns and challenges," International Journal of Green Energy, vol. 12, no. 6, pp. 595-602, 2015.

[5] X. Miao and Q. Wu, "High yield bio-oil production from fast pyrolysis by metabolic controlling of Chlorella protothecoides," Journal of Biotechnology, vol. 110, no. 1, pp. 85-93, 2004.

[6] Z.-Y. Liu, G.-C. Wang, and B.-C. Zhou, "Effect of iron on growth and lipid accumulation in Chlorella vulgaris," Bioresource Technology, vol. 99, no. 11, pp. 4717-4722, 2008.

[7] W. Xiong, X. Li, J. Xiang, and Q. Wu, "High-density fermentation of microalga Chlorella protothecoides in bioreactor for microbio-diesel production," Applied Microbiology and Biotechnology, vol. 78, no. 1, pp. 29-36, 2008.

[8] L. Rodolfi, G. C. Zittelli, N. Bassi et al., "Microalgae for oil: strain selection, induction of lipid synthesis and outdoor mass cultivation in a low-cost photobioreactor," Biotechnology and Bioengineering, vol. 102, no. 1, pp. 100-112, 2009.

[9] A. Vonshak, G. Torzillo, J. Masojidek, and S. Boussiba, "Suboptimal morning temperature induces photoinhibition in dense outdoor cultures of the alga Monodus subterraneus (Eustigmatophyta)," Plant, Cell and Environment, vol. 24, no. 10, pp. 1113-1118, 2001.

[10] N. R. Moheimani and M. A. Borowitzka, “The long-term culture of the coccolithophore Pleurochrysis carterae (Haptophyta) in outdoor raceway ponds," Journal of Applied Phycology, vol. 18, no. 6, pp. 703-712, 2006.

[11] R. Rippka, J. Deruelles, J. B. Waterbury, M. Herdman, and R. Y. Stanier, "Generic assignments, strain histories and properties of pure cultures of cyanobacteria," Journal of General Microbiology, vol. 111, no. 1, pp. 1-61, 1979.

[12] M. A. Saghai-Maroof, K. M. Soliman, R. A. Jorgensen, and R. W. Allard, "Ribosomal DNA spacer-length polymorphisms in barley: mendelian inheritance, chromosomal location, and population dynamics," Proceedings of the National Academy of Sciences of the United States of America, vol. 81, no. 24, pp. 80148018, 1984. 
[13] J. Sambrook, E. F. Fritsch, and T. Maniatis, Molecular Cloning, A Laboratory Manual, Cold Spring Harbor Laboratory Press, 2nd edition, 1989.

[14] C. Bigogno, I. Khozin-Goldberg, S. Boussiba, A. Vonshak, and Z. Cohen, "Lipid and fatty acid composition of the green oleaginous alga Parietochloris incisa, the richest plant source of arachidonic acid," Phytochemistry, vol. 60, no. 5, pp. 497-503, 2002.

[15] C.-H. Hsieh and W.-T. Wu, "Cultivation of microalgae for oil production with a cultivation strategy of urea limitation," Bioresource Technology, vol. 100, no. 17, pp. 3921-3926, 2009.

[16] J. F. Sánchez, J. M. Fernández-Sevilla, F. G. Acién, M. C. Cerón, J. Pérez-Parra, and E. Molina-Grima, "Biomass and lutein productivity of Scenedesmus almeriensis: influence of irradiance, dilution rate and temperature," Applied Microbiology and Biotechnology, vol. 79, no. 5, pp. 719-729, 2008.

[17] Q. Hu, M. Sommerfeld, E. Jarvis et al., "Microalgal triacylglycerols as feedstocks for biofuel production: perspectives and advances," The Plant Journal, vol. 54, no. 4, pp. 621-639, 2008.

[18] I. Khozin-Goldberg and Z. Cohen, "The effect of phosphate starvation on the lipid and fatty acid composition of the fresh water eustigmatophyte Monodus subterraneus," Phytochemistry, vol. 67, no. 7, pp. 696-701, 2006.

[19] G. Huang, F. Chen, D. Wei, X. Zhang, and G. Chen, "Biodiesel production by microalgal biotechnology," Applied Energy, vol. 87, no. 1, pp. 38-46, 2010. 

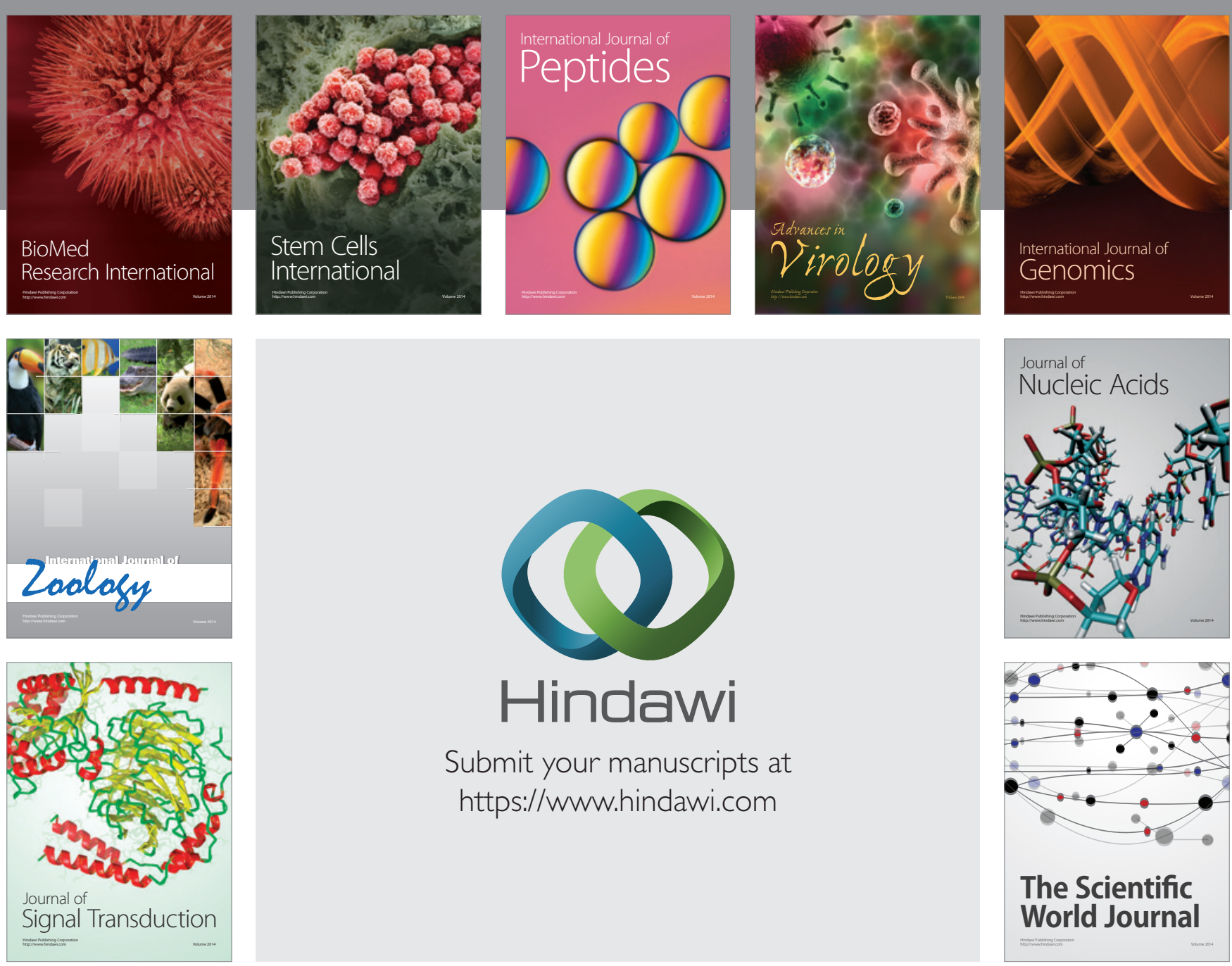

Submit your manuscripts at

https://www.hindawi.com
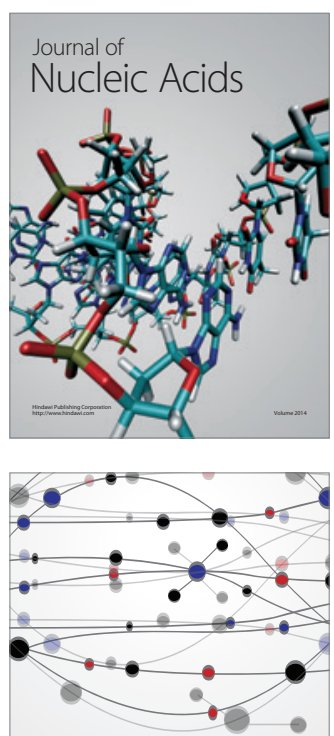

The Scientific World Journal
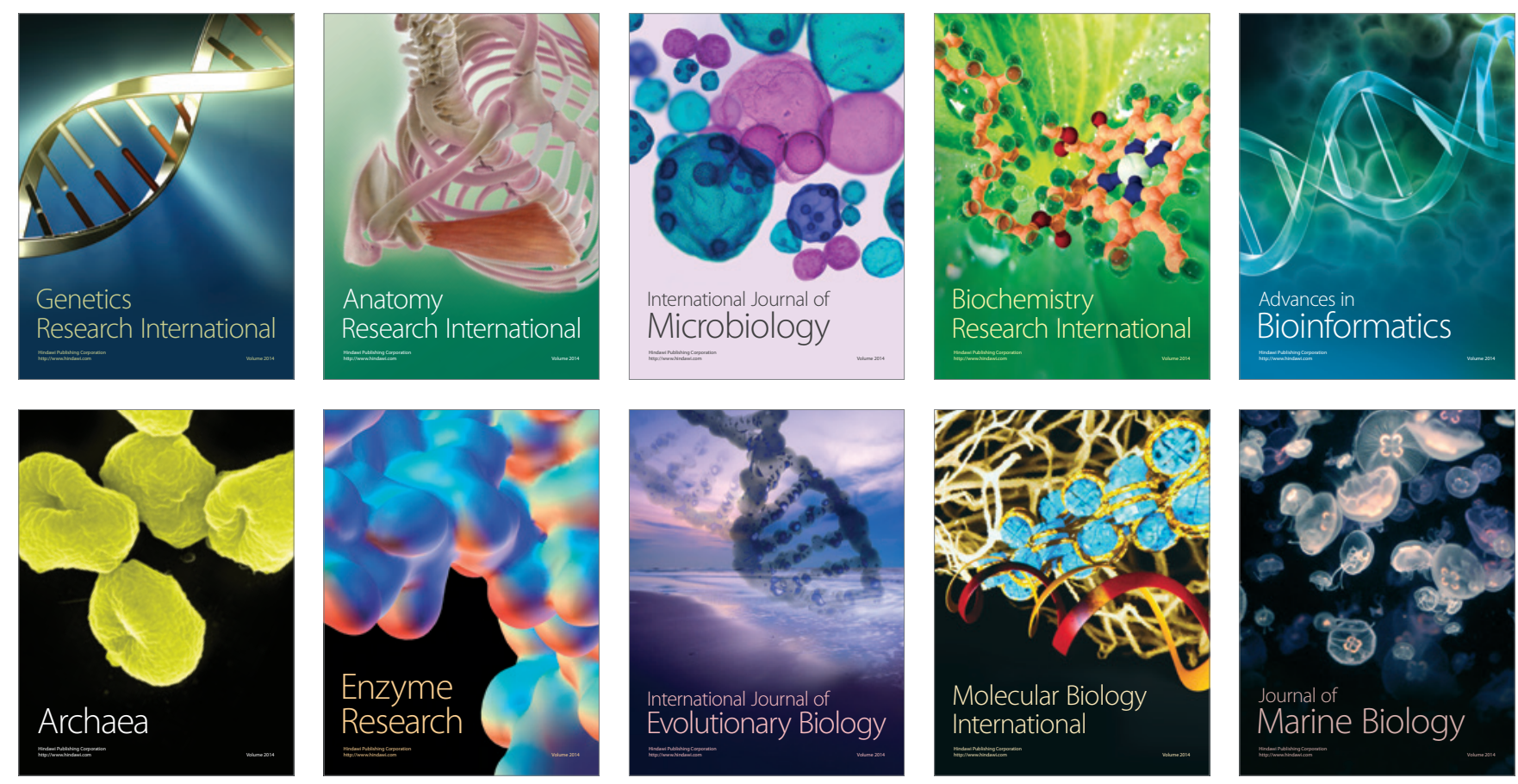